BULL. AUSTRAL. MATH. SOC.

05010,05099

VOL. $24(1981), 289-294$.

\title{
ON A CHARACTERIZATION OF PLANAR GRAPHS
}

\author{
C.C. CHen
}

By introducing the concept of a polygon-extension of a planar graph, we provide a simple proof that a graph is planar if and only if every strict elegant ring in the graph is even.

\section{Introduction}

Throughout, we consider undirected graphs on a finite set of vertices. Following the notations used in [3], we denote the vertex set of a graph $G$ by $V G$ and its edge set by $E G$. If $G$ is a directed graph, $C$ is a directed circuit of $G$, and $a, b$ are distinct vertices of $V C$, then we used the notation $C(a, b)$ to mean the direct subpath of $C$ with origin $a$ and terminus $b$. If $a=b$, then $C(a, b)$ means the subpath of $c$ with vertex set $\{a\}$ and empty edge set. If $P$ is a path in $G$ with end vertices $c, d$, then we use IP to denote the set $V P-\{c, d\}$.

Further, let $X$ and $Y$ be distinct paths or circuits of a graph $G$ with $|V X \cap V Y| \geq 2$. Then an $\bar{X} Y$-path is a maximal nondegenerate subpath $P$ of $Y$ for with $I P \cap V X=\varnothing$ and $E P \cap E X=\emptyset$. An $X Y$-path is a maximal subpath $P$ of $Y$ for which $E P \subseteq E X \cap E Y$ and $V P \subseteq V X \cap V Y$.

Let $S$ be a collection of circuits of $G$. If the edges of $G$ can be directed so that every circuit of $S$ is a directed circuit, then we say that $S$ is consistently orientable. The cyclic sequence of circuits $S=\left(c_{0}, c_{1}, \ldots, c_{n-1}\right)$ with $n \geq 3$ is a ring in the graph $G$, if

(RI) $S$ is consistently orientable,

(R2) $E C_{i} \cap E C_{j} \neq \varnothing$ if and only if $i=j, i \equiv j+1(\bmod n)$

Received 22 April 1981. 


$$
\text { or } i \equiv j-1(\bmod n) \text {, }
$$

(R3) no edge of $G$ belongs to more than two circuits of $G$. We note that (R2) implies (R3) except when $n=3$.

$$
\text { A ring } S=\left(C_{0}, C_{1}, \ldots, C_{n-1}\right) \text { with } n \text { circuits is called an }
$$
$n$-ring. It is called an odd ring if $n$ is odd, an even ring if $n$ is even, a strict ring if $\left|V C_{i} \cap V C_{j}\right| \leq 1$ whenever $E C_{i} \cap E C_{j}=\emptyset$, an elegant ring if, for each $i=0,1, \ldots, n-1$, there is a unique $\bar{c}_{i} c_{i+1}$ path (or equivalently, the common vertices on $C_{i}$ and $C_{i+1}$ are those on the path $M_{i}$ where $\left.E C_{i} \cap E C_{i+1}=E M_{i}\right)$, a perfect ring if it is elegant and $V C_{i} \cap V C_{j}=\emptyset$ whenever $E C_{i} \cap E C_{j}=\emptyset$. We note here, and throughout this paper, that all subscripts are taken as being modulo $n$.

Let $P(G)$ be a planar embedding of a planar graph $G$. For each vertex $x$ of $G$, we shall denote by $N(x)$ the set of all vertices $y$ of $G$ adjacent to $x$ and $E(x)$ the set of all edges of $G$ with one end at $x$. Let $e_{1}, e_{2} \in E(x)$ (say $e_{1}=\left\{x, y_{1}\right\}, e_{2}=\left\{x, y_{2}\right\}$ ). We say that $e_{1}, e_{2}$ are neighbouring edges in $P(G)$ if and only if we can draw a curve $C$ in the plane joining $y_{1}$ and $y_{2}$ such that $C$ does not intersect any $e$ in $E(x)$ except possibly at $y_{1}$ and $y_{2}$ and the open region in the plane bounded by $C, e_{1}, e_{2}$ is disjoint from $N(x)$. We now construct a graph $P^{*}(G)$ whose vertices are all ordered pairs $(x, e)$ with $x \in V G$, $e \in E(x)$ and, for any two vertices $(x, e),(y, f)$ in $V P^{*}(G)$, we draw an edge joining them if and only if one of the following holds:

$$
\begin{aligned}
& \text { (i) } x=y \text { and } e, f \text { are neighbouring; } \\
& \text { (ii) } x \neq y \text { and } e=f \text {. }
\end{aligned}
$$

We shall call $P^{*}(G)$ the polygon extension of $G$ with respect to the planar embedding $P(G)$. Intuitively speaking, $P^{*}(G)$ is obtained from $P(G)$ by replacing each vertex of degree $n$ in $P(G)$ with an $n$-gon and joining corresponding vertices as indicated in the figure below (for $n=4$ ). Note that $P^{*}(G)$ is always planar and if each vertex of $G$ has degree greater than or equal to 3 , then $P^{*}(G)$ is always a cubic graph. 

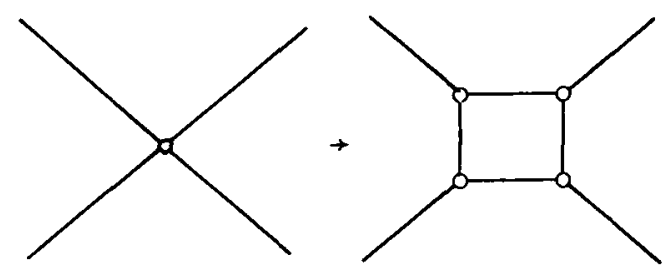

The purpose of this paper is to make use of the concept of polygon extensions of planar graphs to provide a simple proof of the following nice characterization of planar graphs due to Holton and Little [3].

MAIN THEOREM. A groph $G$ is planar if and only if every strict elegant ring in $G$ is even.

REMARK. Throughout this paper, whenever $S$ denotes a ring in a graph $G$, we assume without loss of generality that each edge of $G$ is also an edge of a circuit $C_{i}$ of $S$, since we are concerned only with the subgraph of $G$ which is the union of all circuits of $S$.

\section{Basic lemmas}

The following results will be useful in the sequel.

LEMMA 1 ([1], Kuratowski's Theorem). $G$ is planar if and only if no subgraph of $G$ is homeomorphic to $K_{5}$ or $K_{3,3}$.

LEMMA 2 ([2]). There exists an odd strict elegant ring in $K_{5}$ and in $K_{3,3}$.

Let $C$ be a circuit in the plane. We shall write $I(C)$ to mean the open region in the plane bounded by $C, \overline{I(C)}$ to mean the closed region bounded by $C$, and $O(C)$ to mean the region consisting of all points not in $\overline{I(C)}$.

LEMMA 3. Let $S=\left(C_{0}, C_{1}, \ldots, c_{n-1}\right)$ be a strict elegant ring in a planar graph $G$ and $P(G)$ a planar embedding of $G$. Then, for each $i$, either $I\left(C_{i}\right) \cap V P(G)=\emptyset$ or $O\left(C_{i}\right) \cap V P(G)=\emptyset$.

Proof. Without loss of generality, we need only to consider the case $i=0$. Since $S$ is elegant, either $I\left(C_{0}\right) \cap V C_{1}=\varnothing$ or 
$O\left(c_{0}\right) \cap V C_{1}=\emptyset$ (say the former).

CLAIM. $\quad I\left(C_{0}\right) \cap V C_{2}=\emptyset$.

Indeed, let $e=\{a, b\}$ be a common edge of $C_{1}$ and $C_{2}$. Since $S$ is strict, either $a \notin V C_{0}$ or $b \notin V C_{0}$ (say the former). Hence, we must have $a \in O\left(c_{0}\right) \cap V C_{2}$. Suppose to the contrary that $I\left(C_{0}\right) \cap V C_{2} \neq \emptyset$, say $c \in I\left(C_{0}\right) \cap V C_{2}$. Then $C_{2}(c, a) \cap V C_{0} \neq \emptyset$ and $C_{2}(a, c) \cap V C_{0} \neq \emptyset$. This however contradicts the fact that $S$ is strict and elegant. Thus, we must have $I\left(C_{0}\right) \cap V C_{2}=\emptyset$, as claimed.

By exactly the same argument as that for the above claim, we have $I\left(C_{0}\right) \cap V C_{i}=\emptyset$ for all $i=1,2, \ldots, n-1$. Therefore $I\left(C_{0}\right) \cap V P(G)=\varnothing$, completing the proof.

LEMMA 4. Let $S=\left(c_{0}, c_{1}, \ldots, c_{n-1}\right)$ be a strict elegant ring in a planar graph $G$ and $P(G)$ be a planar embedding of $G$. Then, for each $i$, all adjacent edges in $C_{i}$ are also neighbouring edges in $P(G)$.

Proof. Let $e_{1}=\left\{a, b_{1}\right\}, e_{2}=\left\{a, b_{2}\right\}$ be two adjacent edges of $C_{i}$. By Lemma 3, either $I\left(C_{i}\right) \cap V P(G)=\emptyset$ or $O\left(C_{i}\right) \cap V P(G)=\emptyset$. In the first case, we let $C$ be any curve lying entirely within $I\left(C_{i}\right)$ except for the two ends $b_{1}, b_{2}$ of $c$; where in the second case, we let $C$ be any curve lying entirely within $O\left(C_{i}\right)$ except for the two ends $b_{1}, b_{2}$ of $c$. Then the open region in the plane bounded by $c, e_{1}, e_{2}$ is disjoint from $N(a)$. Thus, by definition, $e_{1}$ and $e_{2}$ are neighbouring edges, as required.

LEMMA 5 ([3]). Let $S=\left(C_{0}, c_{1}, \ldots, C_{n-1}\right)$ be a perfect ring in a planar graph $G$. Then $S$ is even.

Proof. Here as in [3], for each $i$, we shall denote the origin of the unique $\bar{C}_{i} C_{i+1}$-path by $v_{i}$ and the terminus by $u_{i}$. We shall also denote the path $C_{i}\left(u_{i}, v_{i}\right)$ by $P_{i}$. Note that $P_{i}$ is a $C_{i} C_{i+1}$-path. Suppose to the contrary that $S$ is not even. Then we have the following 
two cases to consider.

CASE 1. $n=3$.

In this case, let $e_{1}$ be the edge of $P_{0}$ incident on $v_{0}$, let $e_{2}$ be the other edge of $c_{0}$ incident on $v_{0}$ and let $e_{3}$ be the other edge of $C_{1}$ incident on $v_{0}$. Thus $e_{1} \notin E C_{2}$, and $e_{2}, e_{3}$ cannot both belong to $E C_{2}$. Thus if $v_{0} \in V C_{2}$ then the degenerate path with vertex set $\left\{v_{0}\right\}$ is either a $C_{0} C_{2}$-path or a $C_{1} C_{2}$-path. Since there must be a nondegenerate such path, the elegance of $S$ is contradicted. Thus $v_{0} \neq V C_{2}$ and similarly $u_{0} \notin V C_{2}$. It is now immediate that $C_{0} \cup C_{1} \cup C_{2}$ is a subdivision of $K_{3,3}$, a contradiction to the planarity of $G$.

CASE 2. $n \geq 5$.

In this case, the graph

$$
\begin{aligned}
\bigcup_{k=0}^{n-2}\left[c_{k+1}\left(v_{k}, u_{k+1}\right) \cup c_{k+1}\left(v_{k+1}, u_{k}\right)\right] \cup c_{0}\left(v_{n-1}, u_{0}\right) & \cup c_{0}\left(v_{0}, u_{n-1}\right) \cup P_{0} \cup P_{1} \cup P_{2}
\end{aligned}
$$

is a subdivision of $K_{3,3}$, again a contradiction to the planarity of $G$.

The proof of Lemma 5 is therefore complete.

\section{The proof}

If a graph $G$ is non-planar, by Lemma 1, it contains a subgraph homeomorphic to $K_{5}$ or $K_{3,3}$. Hence, by Lemma $2, G$ contains an odd strict elegant ring.

Conversely, assume that $G$ is planar. Let $s=\left(c_{0}, c_{1}, \ldots, c_{n-1}\right)$ be any strict elegant ring in $G$. We need only to prove that $S$ is even. First, let $P(G)$ be a planar embedding of $G$ and $P^{*}(G)$ the polygonextension of $G$ with respect to $P(G)$. For each $i$ and each $x$ in $V C_{i}$, we denote by $x^{*}$ the set $\{(x, e),(x, f)\}$ where $e, f$ are the two edges of $C_{i}$ incident on $x$. Let $C_{i}^{*}=U\left(x^{*} \mid x \in V C_{i}\right)$. Then, by Lerma 4, $C_{i}^{*}$ forms a circuit in $P^{*}(G)$. Let $S^{*}=\left(C_{0}^{*}, C_{1}^{*}, \ldots, C_{n-1}^{*}\right)$. 
Evidently, $S^{*}$ is a ring in $P^{*}(G)$. The elegance of $S^{*}$ follows immediately from that of $S$. We shall show that $S^{*}$ is a perfect ring. Indeed, let $C_{i}^{*}, C_{j}^{*}$ be any two circuits in $S^{*}$ with $E C_{i}^{*} \cap E C_{j}^{*}=\emptyset$. Then $E C_{i} \cap E C_{j}=\varnothing$. If $V C_{i} \cap V C_{j}=\emptyset$, then it follows from our constructions that $V C_{i}^{*} \cap V C_{j}^{*}=\emptyset$. On the other hand, if $V C_{i} \cap V C_{j}$ is not empty, then, by the strictness of $S$, it must be a singleton. Let $V C_{i} \cap V C_{j}=\{x\}$. Now if $V C_{i}^{*} \cap V C_{j}^{*} \neq \emptyset$, let $(y, e) \in V C_{i}^{*} \cap V C_{j}^{*}$. Then, $y \in V C_{i} \cap V C_{j}$ and so $y=x$. Also $e \in E(x) \cap E C_{i} \cap E C_{j} \subseteq E C_{i} \cap E C_{j}=\emptyset$, a contradiction. Thus in any case, we must have $V C_{i}^{*} \cap V C_{j}^{*}=\emptyset$, showing the $S^{*}$ is a perfect ring. Since $P^{*}(G)$ is planar, by Lemma $5, S^{*}$ is even (that is, $n$ is even). Hence $S$ is even, as required.

\section{References}

[1] Casimir Kuratowski, "Sur le problème des courbes gauches en topologie", Fund. Math. 15 (1930), 271-283.

[2] Charles H.C. Lit+le, "A conjecture about circuits in planar graphs", Combinatorial Mathematics III, 171-175 (Lecture Notes in Mathematics, 452. Springer-Verlag, Berlin, Heidelberg, New York, 1975).

[3] D.A. Holton and C.H.C. Little, "Elegant odd rings and non-planar graphs", Proceedings of the Eighth Australian Conference in Combinatorial Mathematics (to appear).

Department of Mathematics, National University of Singapore, Bukit Timah Road, Singapore 1025, Republic of Singapore. 